\title{
Should We Nebulize Hypertonic Saline Prophylactically in Our Pediatric Intubated Patients?
}

Mucoactive agents improve airway clearance by different mechanisms. According to their mode of action, they can be classified as mucolytics, expectorants, mucokinetics, and ion-transport modifiers. Whereas mucolytic agents disrupt the structure of the mucus gel, thereby reducing its viscosity and elasticity, expectorants promote osmotic passage of water to the airway surface. Mucokinetic drugs improve cough-mediated clearance by reducing mucus adhesivity or by increasing air flow. ${ }^{1}$

Hypertonic saline enhances mucociliary clearance in patients with obstructive lung diseases as well as normal individuals. ${ }^{2}$ It is also considered a safe and effective therapy for neonatal and pediatric conditions requiring mucociliary clearance, such as viral bronchiolitis, ${ }^{3}$ atelectasis, ${ }^{4}$ and cystic fibrosis. ${ }^{5}$ Although hypertonic saline has been traditionally classified as a mucokinetic drug, it has mucolytic properties. Hypertonic saline is capable of disrupting ionic bonds within the mucus gel, changing the rheological properties of the mucus and ultimately reducing its viscosity. ${ }^{1}$ Furthermore, hypertonic saline dissociates DNA from the mucoprotein, allowing natural proteolytic enzymes to digest the mucoprotein. ${ }^{6}$ As a mucokinetic, hypertonic saline increases the liquid on the epithelial surface by osmotically drawing additional water from the mucosa and submucosa into the airway, which hence accumulates in the mucus layer. ${ }^{7}$ The release of prostaglandins observed with the use of hypertonic saline appears to enhance ciliary motility and water absorption from the airway mucosa and submucosa. ${ }^{8}$ Radioaerosol studies in vitro have confirmed the positive effect of hypertonic saline on mucociliary transport and improvement of clearance with cough. ${ }^{9}$ Murray $^{9}$ reported that hypertonic saline was associated with an in vitro reduction of biofilm formation by Pseudomonas aeruginosa and the production of associated virulence factors. In addition to the well-known effects of hypertonic saline, it has been found that it may increase

\footnotetext{
The authors have disclosed no conflicts of interest.

Correspondence: Ruben D Restrepo MD RRT FAARC, Department of Respiratory Care, University of Texas Health Science Center at San Antonio, MSC 6248, San Antonio, TX 78229. E-mail: restrepor@uthscsa.edu.
}

DOI: $10.4187 /$ respcare. 04812 the levels of 2 thiols that are considered protective against oxidative injury, glutathione and thiocyanate, in the airway surface liquid ${ }^{10}$ and may also attenuate lung injury by exerting an anti-inflammatory effect on the pulmonary epithelium. ${ }^{11}$ In the clinical setting, nebulized hypertonic saline, alone or in combination, has been associated with decreased hospital stay and improvement of respiratory severity scores. ${ }^{12}$ Although the use of a bronchodilator takes into account the potential for hypertonic saline to cause irritation of the airway and even bronchospasm in selected patients, a low rate of adverse events has been reported, suggesting that such a combination may be unnecessary. ${ }^{13}$

\section{See the Original Study on Page 586}

In light of the efficacy that has been credited to hypertonic saline as a mucoactive agent, it seems logical to believe that its use in mechanically ventilated patients could provide an additional benefit in the critical care setting. Patients undergoing mechanical ventilation are often at risk for impairment of mucociliary function. Ineffective cough, muscle weakness, suboptimal heat and humidification of medical gases, and a negative fluid balance are some of the contributing factors that lead to inspissation of secretions. In children, the smaller airway size exponentially increases airway resistance and the risk for obstruction and undesired clinical outcomes. Airway obstruction in the mechanically ventilated patient increases the rate of atelectasis and abnormalities in gas exchange, thus increasing the risk for extended stays in the ICU.

A considerably large number of clinical trials and metaanalyses have published the efficacy of mucoactive agents in clinical conditions associated with mucus clearance impairment. ${ }^{14,15,18}$ Nevertheless, the most recent meta-analysis evaluating the therapeutic and prophylactic nebulization of mucoactive agents in adult critically ill patients found very low quantity and quality of evidence to support their use. ${ }^{14}$ Little evidence exists of their prophylactic use in the pediatric critical care setting to decrease the duration of mechanically ventilation, and no prospective blinded studies in children undergoing mechanical ventilation have been reported. 
In this issue of Respiratory CARe, Shein et al ${ }^{15}$ conducted a blind randomized pilot study on the use of hypertonic saline in mechanically ventilated subjects. They investigated the use of nebulized hypertonic saline to improve airway clearance and shorten the duration of mechanical ventilation. They studied 18 children $<18$ y old who had been intubated for $<12 \mathrm{~h}$ and had an expected duration of mechanical ventilation of $>48$ additional $h$. They were prophylactically given $3 \mathrm{~mL}$ of either nebulized hypertonic saline or placebo ( $0.9 \%$ saline $) 4$ times/d. The primary outcome was duration of mechanical ventilation. Ventilator parameters and the presence of wheezing were recorded before and after study drug administration. They found that the duration of mechanical ventilation was significantly longer in children treated with hypertonic saline (208.1 [interquartile range 136.3-319.8] h) versus those treated with placebo (129.5 [interquartile range 74.4-146.1] h) $(P=.03)$. After adjusting for baseline levels of PEEP, the duration of mechanical ventilation did not differ between groups. Mechanical ventilation parameters, including dead space and dynamic compliance, did not differ between measurements taken before study drug administration and measurements taken after. New onset wheezing following study drug administration was rare $(1.0 \%$ with hypertonic saline $[3.0 \%]$ vs placebo, $P=.36$ ). They concluded that prophylactic administration of nebulized hypertonic saline to mechanically ventilated children did not improve clinically relevant outcomes, including duration of mechanical ventilation. In fact, children treated with hypertonic saline had a significantly longer duration of mechanical ventilation. Wheezing after hypertonic saline treatment was rare.

As mentioned by the authors, the study was underpowered, and any generalization of their findings will have to be taken with extreme caution. In addition, children given hypertonic saline had significantly more unfavorable radiographic findings and pulmonary mechanics at enrollment. Having those baseline differences can seriously change the intended outcomes of any therapeutic or prophylactic strategy. An in vitro study by Ari et $\mathrm{al}^{16}$ found that inhaled drug mass can be significantly higher when a manual resuscitation bag is used to administer bronchodilators through an adult artificial airway. However, Schleufe et $\mathrm{al}^{17} \mathrm{did}$ not find any advantage of using Ambu bags to improve aerosol deposition when compared with conventional methods. Assuming that disconnecting patients who have unfavorable radiographic findings, compromised pulmonary mechanics, and high PEEP requirement for the purpose of administering aerosol therapy would not significantly affect clinical outcomes is not easily supported. The disconnection of the patients to bag the treatment should not be considered the standard-of-care method to deliver nebulized hypertonic saline to pediatric patients. Routine disconnection of patients from mechanical venti- lation could increase cross-contamination and may adversely affect duration of ventilation. In addition, the group of subjects with higher PEEP levels in the present study could have experienced alveolar de-recruitment with ventilator disconnection. Although a small group of subjects may not allow generalization of findings, the possibility that those subjects could have been adversely affected by disconnection while receiving hypertonic saline cannot be ruled out. Despite some obvious limitations of the study by Shein et $\mathrm{al}^{15}$, their results are consistent with a few trials where nebulized hypertonic saline did not significantly improve any of the clinical outcomes measured. ${ }^{14,18,19}$

The evidence supporting the prophylactic use of nebulized hypertonic saline for patients undergoing mechanical ventilation as a mucoactive agent and its impact on clinical outcomes is lacking. This study invites clinicians to take a closer look at the potential prophylactic role of mucoactive agents to improve mucociliary clearance and possibly impact important clinical outcomes, such as ventilator length of stay. Only adequately powered clinical studies will reveal the potential role of prophylactic nebulized hypertonic saline in mechanically ventilated pediatric patients.

Ruben D Restrepo MD RRT FAARC Department of Respiratory Care University of Texas Health Science Center at San Antonio San Antonio, Texas

Diana M Serrato MSc CRT Department of Respiratory Care Universidad Santiago de Cali Cali, Colombia and Department of Respiratory Care University of Texas Health Science Center at

San Antonio San Antonio, Texas

\section{REFERENCES}

1. Elkins MR, Bye PT. Mechanisms and applications of hypertonic saline. J R Soc Med 2011;104(Suppl 1):S2-S5.

2. Kellett F, Redfern J, Niven RM. Evaluation of nebulised hypertonic saline $(7 \%)$ as an adjunct to physiotherapy in patients with stable bronchiectasis. Respir Med 2005;99(1):27-31.

3. Badgett RG, Vindhyal M, Stirnaman JT, Gibson CM, Halaby R. A Living systematic review of nebulized hypertonic saline for acute bronchiolitis in infants. JAMA Pediatr 2015;169(8):788-789.

4. Dilmen U, Karagol BS, Oguz SS. Nebulized hypertonic saline and recombinant human DNase in the treatment of pulmonary atelectasis in newborns. Pediatr Int 2011;53(3):328-331.

5. Robinson M, Hemming AL, Regnis JA, Wong AG, Bailey DL, Bautovich GJ, et al. Effect of increasing doses of hypertonic saline on mucociliary clearance in patients with cystic fibrosis. Thorax 1997;52(10):900-903.

6. Lieberman J, Kurnick NB. Influence of deoxyribonucleic acid content on the proteolysis of sputum and pus. Nature 1962;196:988-990. 


\section{Nebulized Hypertonic Saline for Pediatrics}

7. Donaldson SH, Bennett WD, Zeman KL, Knowles MR, Tarran R, Boucher RC. Mucus clearance and lung function in cystic fibrosis with hypertonic saline. N Engl J Med 2006;354(3):241-250.

8. Robinson M, Regnis JA, Bailey DL, King M, Bautovich GJ, Bye PT. Effect of hypertonic saline, amiloride, and cough on mucociliary clearance in patients with cystic fibrosis. Am J Respir Crit Care Med 1996;153(5):1503-1509.

9. Murray TS. The effect of hypertonic saline on in vitro Pseudomonas aeruginosa colonization. Pediatr Pulmol 2010;45:A367.

10. Gould NS, Gauthier S, Kariya CT, Min E, Huang J, Brian DJ. Hypertonic saline increases lung epithelial lining fluid glutathione and thiocyanate: two protective CFTR-dependent thiols against oxidative injury. Respir Res 2010;11:119-120.

11. Wohlauer M, Moore EE, Silliman CC, Fragoso M, Gamboni F, Harr $\mathrm{J}$, et al. Nebulized hypertonic saline attenuates acute lung injury following trauma and hemorrhagic shock via inhibition of matrix metalloproteinase-13. Crit Care Med 2012;40(9):2647-2653.

12. Flores-González JC, Matamala-Morillo MA, Rodríguez-Campoy P, Pérez-Guerrero JJ, Serrano-Moyano B, Comino-Vazquez P, et al. Epinephrine improves the efficacy of nebulized hypertonic saline in moderate bronchiolitis: a randomised clinical trial. PLoS One 2015; 10(11): 0142847.
13. Ralston S, Hill V, Martinez M. Nebulized hypertonic saline without adjunctive bronchodilators for children with bronchiolitis. Pediatrics 2010;126(3):e520-e525.

14. Claudius C, Perner A, Møller MH. Nebulised dornase alfa versus placebo or hypertonic saline in adult critically ill patients: a systematic review of randomised clinical trials with meta-analysis and trial sequential analysis. Syst Rev 2015;4:153-154.

15. Shein SL, Gallagher JT, Deakins KM, Weinert DM. Prophylactic use of nebulized hypertonic saline in mechanically ventilated children: a randomized blinded pilot study. Respir Care 2016;61(5):586-592.

16. Ari A, Harwood RJ, Sheard MM, Fink JB. An in vitro evaluation of aerosol delivery through tracheostomy and endotracheal tubes using different interfaces. Respir Care 2012;57(7):1066-1070.

17. Schleufe P, Reiffen HP, Piepenbrock S. Effective application of bronchodilator aerosols from metered-dose inhalers (MDI) via resuscitator-bag and adapter. Resuscitation 1998;39(3):175-178.

18. Florin TA, Shaw KN, Kittick M, Yakscoe S, Zorc JJ. Nebulized hypertonic saline for bronchiolitis in the emergency department: a randomized clinical trial. JAMA Pediatr 2014;168(7):664-670.

19. Principi T, Komar L. A critical review of "a randomized trial of nebulized 3\% hypertonic saline with epinephrine in the treatment of acute bronchiolitis in the emergency department." J Popul Ther Clin Pharmacol 2011;18(2):e273-e274. 